\title{
Theme: General Pediatrics
}

\section{Levetiracetam monotherapy for childhood epilepsy (Mymensingh Med J. 2018:27:776-84)}

Levetiracetam has been introduced for the control of seizures as monotherapy in children with epilepsy. This randomized controlled trial, conducted in children aged 1 mo to $15 \mathrm{y}$, was done to assess the efficacy and tolerability of levetiracetam compared to phenobarbital in childhood epilepsy (idiopathic focal, generalized, focal with secondary generalization). The children were followed up for 12 months at 3-monthly interval to compare the seizure remission and side effects with Levetiracetam and Phenobarbital. During 3 months of intervention 55.8\% patients of levetiracetam group achieved $50-75 \%$ seizure remission compared to $44.2 \%$ in phenobarbital group; at 6 months, $75-100 \%$ seizure remission observed among $57.4 \%$ patients of levetiracetam group compared to $42.6 \%$ of phenobarbital group, which continued to increase at 9 months in levetiracetam compared to phenobarbital. No further improvement was observed at 12month follow-up. Behavioral problem was reported among 4 patients of phenobarbital group without any evidence of cognitive deterioration; 3 patients receiving levetiracetam experienced irritability, but no child in either treatment group discontinued treatment due to side effects. It appears monotherapy with levetiracetam is more effective in controlling seizures in epilepsy (focal, generalized and focal with secondary generalization) compared to phenobarbital, with minimum side effects.

\section{Chronic cough in under-five children (Medicine.} 2019;98:e13910)

Chronic cough is one of the most common clinical symptoms in children. Its etiology often involves the disciplines of pulmonology, gastroenterology, ENT (ear, nose, and throat), and neurology. Finding the etiology of nonspecific chronic cough is more difficult in under-five children as majority of examinations (such as lung function, induced sputum test, exhaled nitric oxide test) are difficult to perform for this age group. This study attempted to analyze the etiology of nonspecific chronic cough in children under 5 years of age. The medical records of children less than 5 years of age who visited Outpatient Department with cough as the main clinical manifestation of more than 4 weeks and no significant abnormalities in the chest $X$-ray film were included. Exclusion criteria included children having other symptoms besides cough, abnormal chest $X$-ray and serious systemic diseases. Upper airway cough syndrome was the most common (37.6\%) cause of nonspecific chronic cough, followed by cough variant asthma (31.8\%) and post-infection cough (18.8\%).

\section{Physical activity in adolescents and Quality of life (Health Qual Life Outcomes. 2019;17:2)}

Health-related quality of life is a multidimensional concept with significant effects on children and adolescents. Physical activity and screen time have been suggested as its probable predictors.
This study aimed to investigate the association of physical activity, screen time and their combination with health-related quality of life in a sample of Iranian children and adolescents. A sample of 25,000 students aged 6-18 years was selected via multistage cluster sampling from 30 provinces of Iran. Sociodemographic data was obtained by using the questionnaire of the World Health Organization-Global school-based student health survey (GSHS). Persian Pediatric Quality of Life inventory (PedsQL) and Physical Activity Questionnaire for the pediatric age group (PAQ-A) were applied for evaluating health related quality of life and physical activity, respectively. Physical activity scores 1-1.9 and 2-5 were respectively considered as low and high. The average duration of time spent on watching TV and leisure time computer use were considered as screen time behaviors. Screen time of less than 2 hours was considered low. Screen time duration had an inverse relationship with quality of life while physical activity was associated with better quality of life. High physical activity and low screen time had the best health related quality of life scores.

Thus it is essential to increase play time and other outdoor activities for children and adolescents to improve quality of life, especially in cities with lack of open spaces and playgrounds.

Papaya seeds for deworming. (BMC Complement Altern Med. 2018;18:327)

Soil transmitted helminths (STH) are among the world's neglected tropical diseases. Morbidity due to STH is greatest in school-age children who typically have the highest burden of infection. In this study, maize (corn) flour fortified with grounded dried papaya (Carica papaya) seeds was used to prepare porridge for three primary schools in Kenya. One school received $300 \mathrm{~mL}$ papaya fortified porridge daily (papaya group), the second school received similar serving of plain porridge without the papaya ingredient (control group), and the third school received plain porridge and the conventional deworming agent $400 \mathrm{mg}$ albendazole. The children were monitored daily for two months and final stool sample analysis and clinical monitoring was done at the end of the study. The overall prevalence of Ascaris lumbricoides was $29.4 \%$, Trichuris trichura $5.2 \%$, and hookworm $1 \%$. After two months, papaya seed fortified porridge reduced the Ascaris egg count by $63.9 \%$ as compared to $78.8 \%$ in the albendazole arm. The control group showed an increase in egg count; though, it was not statistically significant. Hemoglobin counts in the papaya group increased by a mean of $2 \mathrm{~g} / \mathrm{dL}$ as compared to the albendazole arm where it increased by $1 \mathrm{~g} / \mathrm{dL}$. No significant change was observed in the placebo arm. Interestingly, the papaya group showed a significant reduction of children with Tinea capitis (ringworms) as compared to the albendazole arm that showed an increase in ringworm infestation.

K RAJESHWARI drkrajeshwari@hotmail.com 